\title{
Licht am Ende des Tunnels? Der Aufbau der Afghanischen Nationalarmee
}

\author{
Michael Paul*
}

\begin{abstract}
Public support for the ISAF mission in troop-contributing countries is falling. Thus, NATO's Secretary General Rassmussen declared that "our populations, Afghan and international, want to see light at the end of the tunnel." Will the Afghan National Army (ANA) shed some light on the war in Afghanistan? Part of the problem is that the Bush administration switched its focus from Afghanistan to Iraq in 2002, a priority only recently reversed. Of the 86 ANA battalions, or kandaks, fielded to date, 46 are rated capable of operating independently. But the emphasis on ANA infantry forces means that NATO forces have to provide everything else, from artillery and air strikes to medical care and supplies. For the ill-equipped ANA, advisers are a lifeline to the enormous resources of NATO. In a shooting war where a weak indigenous force faces a threat beyond its capabilities, a training effort can complement a larger NATO military presence but not substitute for one. The ANA is on the right way, but it will take strategic patience and endurance to build up an ability to substitute NATO forces
\end{abstract}

Keywords: Afghanistan, NATO, ANA, training

Afghanistan, NATO, ANA, Ausbildung

Im Koalitionsvertrag der Bundesregierung wird das Engagement in Afghanistan als eine Aufgabe von besonderem nationalen Interesse bezeichnet; denn es diene deutschen Sicherheitsinteressen, sei Ausdruck der Solidarität mit den Menschen in Afghanistan und bekräftige die deutsche Verlässlichkeit als NATO-Mitglied. ${ }^{1}$ Ein unbegrenztes deutsches Engagement ist jedoch ebenso klar nicht im nationalen Interesse. Daher hat Bundeskanzlerin Angela Merkel bereits in ihrer Regierungserklärung am 8. September 2009 eine zeitliche Ausstiegsperspektive genannt: Innerhalb der nächsten fünf Jahre müssen „substanzielle, qualitative Fortschritte“ erzielt werden, die es der „International Security Assistance Force“ (ISAF) schrittweise ermöglichen sollen, das Engagement zu reduzieren. Vor dem Hintergrund des 2010 zu erneuernden „Afghanistan Compact", der den Rahmen für die Zusammenarbeit festlegt, sollen in einer internationalen Konferenz Ende Januar 2010 vor allem „Zielvorgaben zur Zahl und Qualität der auszubildenden afghanischen Sicherheitskräfte ... einschließlich klarer Zeitvorgaben, in denen dies zu geschehen hat", festgelegt werden. ${ }^{2}$ Neben polizeilichen Kräften betrifft dies den Aufbau der Afghanischen Nationalarmee (ANA), deren Ausbildung die NATO unterstützt. Wie auch US-Präsident Barack Obama in seinen Grundsatzreden über die Afghanistan-Strategie am 27. März und am 1. Dezember 2009 verknüpfte die Bundeskanzlerin verstärkte Ausbildungsmaßnahmen mit einer „Übergabestrategie

* Dr. phil. Michael Paul, Senior Fellow, Forschungsgruppe Sicherheitspolitik, Deutsches Institut für Internationale Politik und Sicherheit, Stiftung Wissenschaft und Politik (SWP), Berlin. Der Beitrag basiert auf einer aktualisierten und überarbeiteten Fassung von Michael Paul, Der (Wieder-) Aufbau der Afghanischen Nationalarmee: Ausweg für die NATO oder Menetekel für Afghanistan? Berlin: SWP, November 2009 (SWP-Aktuell 60/2009).

1 Siehe Koalitionsvertrag zwischen CDU, CSU und FDP, S. 122 http://www.cdu. de/doc/pdfc/091026-koalitionsvertrag-cducsu-fdp.pdf.

2 Regierungserklärung und Debatte über den Bundeswehreinsatz in Afghanistan, 233. Sitzung des 16. Deutschen Bundestages am 8. September 2009, S. 26298, http://dip21.bundestag.de/dip21/btp/16/16233.pdf. in Verantwortung“. ${ }^{3}$ Den Plänen der Bundesregierung zufolge sollen afghanische Sicherheitskräfte „frühestmöglich“ befähigt werden, ,zunächst lokal, dann flächendeckend den Schutz der Bevölkerung sowie ziviler Aufbauleistungen in einem gesicherten Umfeld zu gewährleisten“.

Angesichts des geringen Entwicklungsstandes und wachsender Gewalt im Land ist jede Überlegung, wie Verantwortung an die afghanische Regierung übergeben und der ISAF-Einsatz beendet werden kann, längerfristig zu sehen. Eine Exit-Strategie sollte dabei weniger an Zeitachsen ausgerichtet als an die Erfüllung bestimmter Fortschrittskriterien (bench marks) gebunden werden - insofern ist auch das von Obama genannte Datum eines beginnenden Abzugs im Juli 2011 relativ zur konkreten Lage zu sehen. ${ }^{5}$ Denn ein übereilter Abzug würde die erreichten Fortschritte im Staatsaufbau zunichte machen und könnte die Taliban an die Macht zurückbringen. Damit könnten die Ausbildung von Terroristen und der Export des Terrorismus an einer der sensibelsten Schnittstellen der Weltpolitik wieder aufleben. Andererseits ließe sich argumentieren, dass nicht abzuschätzen ist, welche Folgen ein Ende des internationalen Engagements hätte: Zwar werden die Taliban im Süden von Paschtunen unterstützt, doch die Mehrheit der Afghanen dürfte wegen der negativen Erfahrungen mit dem Taliban-Regime (1996 - 2001)

3 Ebd., S. 26299. Vgl. The White House, Remarks by the President on a New Strategy for Afghanistan and Pakistan, 27. März 2009, http://www.whitehouse. gov/the press office/Remarks-by-the-President-on-a-New-Strategy-for-Afghanistan-and-Pakistan/ sowie The White House, Remarks by the President to the Nation on the Way Forward in Afghanistan and Pakistan, 1. Dezember 2009, http://www.america.gov/st/texttrans-english/2009/December/ 20091201214809ptellivremos0.4085003.html.

4 Siehe Bundesregierung, Afghanistan. Auf dem Weg zur „Übergabe in Verantwor tung “, Kabinettsbeschluss vom 18. November 2009, http://www.bundesregierung.de/Content/DE/_Anlagen/2009/11/2009-11-18-dokument-afghanistan ,property=publicationFile.pdf.

5 So erklärte US-Verteidigungsminister Gates: "Our current plan is that we will begin the transition in local areas in July of 2011 ... [But] we will evaluate in December 2010 whether we believe we will be able to meet that objective. If circumstances dictate in December the president always has the freedom to adjust his decisions." Zitiert nach: Daniel Dombey/James Blitz, "US may revise Afghan withdrawal date", Financial Times, 2. Dezember 2009, http:// www.ft.com/cms/s/0/c4efd1d6-df74-11de-98ca-00144feab49a.html?nclick_ check=1. 
keine Illusionen haben und könnte sich gegen deren Rückkehr an die Macht wenden. ${ }^{6}$ Und selbst wenn die Taliban die Macht übernähmen, hieße das nicht zwangsläufig, dass die Terrororganisation Al Kaida erneut an Einfluss gewänne.

Eine der unverzichtbaren Bedingungen auf dem Weg zu selbsttragenden Sicherheitsstrukturen ist der Aufbau afghanischer Sicherheitskräfte, insbesondere der ANA. Wie ist deren Leistungsfähigkeit zu bewerten und welche Perspektiven ergeben sich mittel- und langfristig? Kann mittels einer gut ausgebildeten ANA als Teil des afghanischen Sicherheitsapparats das militärische Engagement der NATO verringert werden?

Grundlegend bietet nur eine Kombination ziviler und militärischer Unterstützungsleistungen für die afghanische Regierung einerseits und die Erfüllung zentraler Forderungen durch die Regierung in Kabul (wie gute Regierungsführung und Korruptionsabbau) andererseits eine realistische Ausstiegsperspektive. Der Aufbau staatlicher Strukturen ist ein langwieriges Unternehmen. Ohne diese zivile Entwicklung ist aber auch eine militärische Großorganisation wie eine Nationalarmee nicht zu beherrschen. Im Mittelpunkt des internationalen Engagements muss daher die Förderung afghanischer Eigenverantwortung stehen. Die Ausbildung afghanischer Sicherheitskräfte sollte verstärkt unterstützt und erreichter Ausbildungsstand sowie Einsatzwert kontinuierlich anhand klarer Kriterien überprüft werden. Dem Primat der Politik folgend sind parallel dazu die politischen Leitungsebenen in Kabul entsprechend zu befähigen, andernfalls drohen Szenarien, in denen militärische Macht missbraucht werden könnte.

\section{Die ANA aus dem Blickwinkel der ISAF-Entsen- destaaten}

Die Förderung des Aufwuchses der ANA - parallel zur Ausbildung polizeilicher Kräfte wie der „Afghan National Police“ (ANP) - ist vor dem Hintergrund der geringen Akzeptanz des Einsatzes in den ISAF-Entsendestaaten zu sehen. Angeführt von Deutschland (63 Prozent) ${ }^{7}$ besteht nach Umfragen vom August 2009 eine signifikante Ablehnung, weitere Truppen nach Afghanistan zu senden, in Frankreich (62 Prozent), Polen (57 Prozent), Kanada (55 Prozent) und Großbritannien (51 Prozent). ${ }^{8}$ Auch in den USA befürworten nur noch 24 Prozent der Befragten die Entsendung zusätzlicher Truppen, während

6 In einer Umfrage von ABC News, ARD und BBC zeigte eine Mehrheit der landesweit im Dezember 2009 befragten Afghanen eine wachsende Antipathie gegenüber den Taliban: 90 Prozent unterstützten die gegenwärtige Regierung verglichen mit sechs Prozent, die eine Taliban-Administration befürworteten. 69 Prozent bezeichneten die Taliban, El Kaida und fremde Kämpfer als größte Gefahr für das Land. Siehe Adam Mynott, „Afghans more optimistic for future, survey shows”, BBC News, 11. Januar 2010, http://news.bbc.co.uk/2/hi/ south asia/8448930.stm.

7 Mittlerweile ist die Zustimmung zum Afghanistan-Einsatz der Bundeswehr gewachsen. Im ARD-DeutschlandTrend sprachen sich 57 Prozent der Bundesbürger dafür aus, dass die Bundeswehr „sich möglichst schnell aus Afghanistan zurückziehen sollte“. Dies waren zwölf Punkte weniger im Vergleich zum Juli 2009. 37 Prozent waren der Meinung, die Bundeswehr sollte weiterhin in Afghanistan stationiert bleiben (zehn Prozentpunkte mehr als im Juli). ARD, „Zustimmung zu Afghanistan-Einsatz gestiegen“, DeutschlandTrend, 10. September 2009, http://www.tagesschau.de/wahl/umfragen/deutschlandtrend860.html.

8 Richard Auxier, Few in NATO Support Call For Additional Forces in Afghanistan, Pew Research Center, 31. August 2009, http://pewresearch.org/pubs/1325/little-support-in-nato-for-afghanistan-troop-increases. etwa doppelt so viele die Truppenzahl verringern würden. ${ }^{9}$ Dementsprechend forderte US-Senator Carl Levin, Vorsitzender des Streitkräfteausschusses, keine weiteren amerikanischen Soldaten einzusetzen, sondern die Ausbildung afghanischer Sicherheitskräfte zu forcieren. Dieser Plan wurde allerdings heftig kritisiert, weil er ausschließlich auf die quantitative Generierung afghanischer Truppen ausgerichtet war. ${ }^{10}$

Grundsätzlich ist die ANA ein wichtiges, aber nicht das zentrale Element eines zivile und militärische Aspekte umfassenden Engagements der internationalen Staatengemeinschaft. Ohne Regierungsstrukturen könnte die Nationalarmee wie schon in den 1980er Jahren erneut in Milizen zerfallen. Selbsttragende Sicherheit und Stabilität zu erzeugen ist aber ohne nationale Streitkräfte unmöglich. Idealiter kann die ANA also nicht nur die bereits stationierten ISAF-Truppen entlasten, sondern mittel- und längerfristig deren Aufgaben übernehmen. Der Aufbau einer unter zivilem Oberbefehl stehenden, personell und materiell hinlänglich ausgestatteten Nationalarmee liegt damit klar im Interesse der NATO. Wie der gesamte ISAF-Einsatz basiert die Ausbildungsunterstützung dabei auf Resolution 1386 (2001) des VN-Sicherheitsrats und folgenden Resolutionen.

\section{Missglückter Start und neuer Anfang}

Befürworter eines raschen Abzugs aus Afghanistan argumentieren oft, die inzwischen achtjährige Anwesenheit der ISAF habe dem Land keinen Frieden gebracht (und übersehen dabei, dass ISAF erst ab Mitte 2006 alle Landesteile umfasste). ${ }^{11}$ Tatsächlich wurde - nicht zuletzt aufgrund des Irakkrieges - erst vor Kurzem damit begonnen, eine der Komplexität des Landes angemessene und den Aufgaben entsprechende Politik zu betreiben. Die ANA ist exemplarisch: Waren 2002 für eine 70.000 Mann umfassende Armee und Grenzschutz 130 Millionen US-Dollar vorgesehen, haben die USA für eine knapp doppelt so große Armee vier Mrd. US-Dollar im Haushaltsjahr 2009 eingeplant. ${ }^{12}$ Erst seit 2007 wurde die ANA so ausgestattet, dass sie eigenständig operieren konnte. Seitdem wurde die Zahl jährlich ausgebildeter Soldaten verdreifacht, so dass die ANA nun 94.000 Mann umfasst. ${ }^{13}$ Wertvolle Zeit wurde also durch ungenügenden Personal- und Ressourceneinsatz vergeudet ${ }^{14}$ - Jahre, welche die Aufstandsbewegung mit den Taliban als stärkster und bestorganisierter Kraft nutzte, um ihre Fähigkeiten zu verbessern.

9 Jennifer Agiesta/Jon Cohen, "Public Opinion in U.S. Turns Against Afghan War", Washington Post, 20.8.2009, http://www.washingtonpost.com/wp-dyn/ content/article/2009/08/19/AR2009081903066_pf.html.

10 Siehe Anthony H. Cordesman, The Levin Plan - A wrong approach in Afghanistan, Washington, D.C.: CSIS, 12. September 2009, http://csis.org/files/publication/090914_Afghan.The\%20Levin\%20Plan.pdf.

11 Siehe NATO's role in Afghanistan, 23. Oktober 2009, http://www.nato.int/cps/ en/natolive/topics_8189.htm\#evolution.

12 Vgl. Obaid Younossi/Peter Dahl Thruelsen/Jonathan Vaccaro/Jerry M. Solinger/Brian Grady, The Long March. Building an Afghan National Army, Santa Monica/CA: RAND National Defense Research Institute, 2009, S. 12; US Department of Defense, Progress toward Security and Stability in Afghanistan. Report to Congress in accordance with the 2008 National Defense Authorization Act, Juni 2009, S. 27.

13 Siehe Ronald Neumann, „Protecting Afghan Civilians“, DefenseNews, 10. August 2009, S. 21 und Meredith Buel, "Pentagon: Afghan Army Growing at Record Pace", VOA News, 12. November 2008.

14 Siehe Anthony H. Cordesman/Adam Mausner/David Kasten, Winning in Afghanistan. Creating Effective Afghan Security Forces, Washington, D.C.: CSIS, Mai 2009, S. 31-34 http://www.humansecuritygateway.nfo/documents/SIS_WinningInAfghanistan_CreatingEffectiveAfghanSecurityForces.pdf. 
Eine weitere Begründung für notwendige Investitionen in die Ausbildung afghanischer Sicherheitskräfte resultiert daher aus der - zum Teil im zivilen ${ }^{15}$ und im militärischen ${ }^{16}$ Kontext selbst verursachten - verschärften Sicherheitslage. Zuletzt warnte der Oberbefehlshaber der US- und NATO-Truppen Stanley McChrystal Ende August 2009 vor einem Scheitern des Afghanistan-Einsatzes, sollte nicht binnen eines Jahres die Zahl der Truppen erhöht werden. Gelinge es nicht innerhalb kurzer Frist, die Initiative zu übernehmen und die Stoßkraft der Aufständischen abzuwenden, drohe eine Lage, in der ein Aufstand nicht länger niedergeschlagen werden könne, meinte McChrystal. Mehr Ressourcen seien zwar keine Garantie für einen Sieg, zu wenige könnten aber zur Niederlage führen. ${ }^{17}$

Auf deutscher Seite wurde das Bundestagsmandat mit einer Obergrenze von bis zu 4.500 Soldatinnen und Soldaten der Bundeswehr im November 2009 beibehalten. Welche Perspektive für das weitere Vorgehen im Rahmen der Londoner Afghanistan-Konferenz am 28. Januar 2010 festgelegt werden wird, ist derzeit noch offen. So muss die Bundesregierung entscheiden, ob und wenn ja, in welcher Anzahl und zu welchem Zweck - beispielsweise im Rahmen verstärkter ANA-Ausbildungsunterstützung - zusätzliche Soldaten der Bundeswehr an den Hindukusch zu entsenden sind. Zwar könnten afghanische Sicherheitskräfte schrittweise Verantwortung für die Sicherheit der Bevölkerung und des Staates übernehmen, dies erfordert zunächst aber einen verstärkten Mittel- und Personaleinsatz durch die ISAF-Entsendestaaten. Eine „international abgestimmte Übergabestrategie in Verantwortung“ (Merkel) könnte dann die Voraussetzung schaffen, die ISAF-Präsenzabzubauen. Angesichts der Bedingungen ist mit einem mehrjährigen Übergangsprozess zu rechnen.

\subsection{Afghanische Rahmenbedingungen}

In Afghanistan zählen persönliche Loyalität, lokale Autorität und Stammestraditionen mehr als nationale Einrichtungen. Wegen der unwegsamen Topografie gilt Kabul für die Mehrheit der Afghanen als Sitz einer abstrakten, weit entfernten Regierung. Die zahlreichen Fälle von Wahlfälschung haben das Ansehen der Zentralregierung geschwächt und eine Legitimitätskrise ausgelöst, von der auch die in Afghanistan tätige

15 Es fehlt afghanischen Institutionen nicht nur an den Voraussetzungen für eine effiziente und finanziell sichere Durchführung der Aufbauhilfe (was zur Bildung von Parallelstrukturen führte, die den Aufbau afghanischer Strukturen schwächen, zumal nur 20 Prozent der Finanzhilfe direkt durch afghanische Regierungsstellen verwaltet werden), sondern die Hilfe beträgt auch weniger als zehn Prozent z.B. der in Bosnien-Herzegowina aufgewendeten Mittel. Zudem wird geschätzt, dass 80 Prozent der Mittel, die als Entwicklungshilfe ausgegeben werden, in das Geberland zurückfließen (Beratungsgebühren, Verträge mit Firmen im eigenen Land, Verwaltungsaufwand). Vgl. Kishore Mahbubani, „Der Mythos westlicher Entwicklungshilfe“, E+Z, Jg. 49, Nr. 2 (2008), S. 69

16 "Even today, seven years into the U.S.-led war effort, NATO, U.S., and Afghan forces don't always coordinate their missions, military experts say, and it's not uncommon for U.S. and Afghan forces operating jointly to have no idea what NATO's ISAF forces are doing and vice versus. One former senior U.S. military commander ... called 'unity of effort' the most serious problem we have in Afghanistan today. 'It's not the Taliban. It's not governance. It's not security,' he said. 'It's the utter failure in the unity of effort department'." Greg Bruno, "Afghanistan's National Security Forces", CFR Backgrounder, 16. April 2009, http://www.cfr.org/publication/19122/afghanistans_national_security_forces.html?breadcrumb $=\% 2 \mathrm{~F}$.

17 Stanley McChrystal, Commander's Initial Assessment, Kabul: HQ ISAF, 30. August 2009. internationale Staatengemeinschaft betroffen ist. ${ }^{18}$ Zudem benötigte Präsident Karsai für seine Bemühungen um eine Wiederwahl die Unterstützung der Gouverneure und ehemaligen Kriegsherren, die damit ihrerseits an der Macht teilhaben. Wie werden sich Gouverneure und lokale Führer zu schlagkräftigen Sicherheitskräften verhalten - von den Nachbarstaaten hier einmal abgesehen ${ }^{19}$ Es ist davon auszugehen, dass staatliche Sicherheitsorgane akzeptiert werden, wenn sie die Sicherheit der Bevölkerung vor den Taliban verbessern, ohne zugleich den Machtanspruch der lokalen bzw. regionalen Autoritäten in Frage zu stellen. Allerdings stellt sich langfristig die Frage nach dem staatlichen Gewaltmonopol. Sollten die afghanischen Sicherheitskräfte eines Tages über durchsetzungsfähige Strukturen verfügen, müssen die weiter bestehenden Milizen aufgelöst oder in staatliche Gefüge - ggf. auf Regionalebene - integriert werden. ${ }^{20}$

Als Vielvölkerstaat bleibt die extreme Fraktionierung der Gesellschaft ein Konfliktherd, doch ist ein gesamtafghanisches Selbstverständnis heute so stark ausgeprägt wie nie zuvor in der Geschichte. Zudem existiert ein historisches Vorbild für die ANA. Vor dem Bürgerkrieg gab es eine professionelle Armee als nationale Institution, die sich aber mit dem Sturz des von Moskau eingesetzten Präsidenten Nadschibullah 1992 endgültig auflöste. ${ }^{21}$ Schon in den 1980er Jahren hatten sich im Widerstand gegen die sowjetischen Truppen lokal, tribal und ethnisch organisierte Milizen formiert, auf die sich später die Mudschaheddin in Kabul (1992-96) und das Taliban-Regime stützten. So besitzt Afghanistan zwar eine lange Armeetradition, eine weit längere Erfahrung gibt es aber mit Stammesmilizen. ${ }^{22}$

Die größte Herausforderung besteht darin, neue staatliche Institutionen zu schaffen, die ethnisch, konfessionell und regional ausgewogen sind. Eine hohe Analphabetenrate und ein niedriger Bildungsstand erschweren die Ausbildung. Eine Armee westlichen Musters soll und wird die ANA auf absehbare Zeit nicht werden. Dies wäre ein unerreichbares Ausbildungsziel und der Ansatz wäre im Sinne einer Übergabestrategie mit einem „afghan face“ auch verfehlt.

\subsection{Die ANA-Ausbildung}

Eine Armee im Sinne afghanischer Eigenverantwortung aufzubauen, erfordert mehr, als eine Hilfstruppe zusammenzustellen. Jedoch waren die anfänglichen Investitionen gering und das Training rudimentär. ${ }^{23}$ Als Leitnation haben die USA im Dezember 2002 die Verantwortung für die Ausbildung, den

18 Vgl. Thomas Ruttig, Afghanistans Wahlkrise, Berlin: SWP, Oktober 2009 (SWPAktuell 2009/56).

19 Vgl. Paul, Der (Wieder-) Aufbau der Afghanischen Nationalarmee, S. 2.

20 Im Januar 2007 sollen über 500 Regierungsvertreter ihre eigene Miliz unterhalten haben oder von einer der Milizgruppierungen unterstützt worden sein. Siehe Vishal Chandra, "Making of the New Afghan National Army: Challenges and Prospects", Strategic Analysis, Vol. 33, No. 1 (Januar 2009), S. 61.

21 Vgl. Chandra, "Making of the New Afghan National Army", S.55.

22 Vgl. Younossi u.a., The Long March., S. 5-7.

23 Vgl. Antonio Giustozzi, "Auxiliary Force or National Army? Afghanistan's ANA and the Counter-Insurgency Effort", in: Small Wars and Insurgencies, Vol. 18, Nr. 1 (2007), S. 48 und S. 55 sowie Cordesman/Mausner/Kasten, Winning in Afghanistan, S.59ff. 
Aufbau und die Ausrüstung übernommen. ${ }^{24}$ Federführend ist auf amerikanischer Seite das „Combined Security Transition Command - Afghanistan” (CSTC-A) unter gemeinsamer Führung mit der auf dem NATO-Gipfeltreffen in Straßburg/Kehl etablierten „NATO Training Mission - Afghanistan“ (NTMA). ${ }^{25}$ Auf afghanischer Seite werden alle Ausbildungsmaßnahmen seit Juli 2006 vom „ANA Training Command“ koordiniert, zu dem unter anderem das „Kabul Military Training Centre“ (KMTC) sowie die Truppenschulen und die Verteidigungsakademie gehören. ${ }^{26}$

Die ANA ist dem seit Dezember 2004 amtierenden Verteidigungsminister Abdul Rahim Wardak unterstellt und besaß im Oktober 2009 einen Personalumfang von 94.000 Mann. Die Zielgröße von 134.000 Soldaten (122.000 Soldaten und 12.000 Ausbildungsplätze) soll im Dezember 2011 - gegebenenfalls sogar bereits zum Oktober $2010^{27}$ - erreicht werden. Damit wurde das im September 2008 festgelegte Datum um zwei bzw. drei Jahre vorverlegt.

Entsprechend den ISAF-Regionalkommandos ist die ANA in fünf regionale Korps sowie ein Heeresfliegerkorps gegliedert. Jedes Korps setzt sich aus drei Brigaden zusammen, die in fünf „Kandaks“ - der paschtunischen Bezeichnung für ein Bataillon - mit je 600 Soldaten organisiert sind. Eine Brigade besteht aus drei Infanterie-Kandaks, einem Kandak zur Kampfunterstützung und einem Logistik-Kandak. Hinzu kommt je ein Kommando-Kandak je Korps, das - in Abstimmung mit dem jeweiligen Regionalkommando - zentral vom Verteidigungsministerium in Kabul geführt wird. Im Oktober 2009 waren 46 von 86 Kandaks zu eigenständigen Operationen fähig und hatten damit das höchste Fähigkeitsprofil (Capability Milestone, CM-1) erreicht; 58 weitere sollen bis Anfang 2010 aufgestellt werden (CM-5). ${ }^{28}$ Auch zwei der fünf Regionalkorps im Osten und Süden (das 203. Korps in Gardez und das 205. Korps in Kandahar) sind voll einsatzfähig. Bei Kampfverbänden hat sich die Rate der unerlaubten Entfernung von der Truppe von 33 Prozent im Jahr 2006 auf neun Prozent im Jahr 2009 verringert. Insofern haben sich personeller Aufwuchs sowie materielle und immaterielle Parameter (von der Bezahlung bis hin zu einem „Korpsgeist“") positiv verändert. ${ }^{29}$

24 Anlässlich der Tokioter Geberkonferenz wurde im Januar 2002 beschlossen, eine umfassende Reform des Sicherheitssektors in Afghanistan zu unterstützen. Die Koordinierungsfunktion für einzelne Bereiche sollten jeweils Führungsnationen übernehmen. So die USA den Aufbau der ANA, Italien den Aufbau des Justizsektors, Großbritannien den Kampf gegen die Opiumökonomie, Japan die Entwaffnung, Demobilisierung und Reintegration ehemaliger Kämpfer sowie Deutschland den Aufbau der Polizei.

25 Siehe NATO, NATO Training Mission - Afghanistan, 4. April 2009, http://www. nato.int/cps/en/natolive/news_52802.htm.

26 Vgl. Chandra, "Making of the New Afghan National Army", S.56.

27 McChrystal, Commander's Initial Assessment, S. G-1

28 Um ein Fähigkeitsprofil der ANA zu erstellen und die Entwicklung zu prognostizieren, werden alle Verbände und Stäbe durch CSTC-A anhand von definierten "Capability Milestones (CM)" bewertet; CM-1 entspricht als höchstes von fünf Profilen der Fähigkeit zu eigenständigen Operationen. Siehe CJ Radin, "Afghan National Army: February 2009 Update", http://www.longwarjournal.org/archives/2010/02/afghan_national_army_1.php. Vgl. Younossi u.a., The Long March, S. 41.

29 Anthony H. Cordesman, Afghan National Security Forces: Shaping the Path to Victory, Washington, D.C.: CSIS, 27. Juli 2009, S. iii; Cordesman/Mausner/ Kasten, Winning in Afghanistan, S. 57 und S. 70; US Department of Defense, Progress toward Security and Stability in Afghanistan, S. 28-31, S. 34; Antonio Giustozzi, "Afghanistan's National Army: The Ambiguous Prospects of Afghanization", Terrorism Monitor, Vol. 6 Nr. 9 (1. Mai 2008), http://www. jamestown.org/single/?no cache=1\&tx ttnews[swords]=8fd5893941d69d0 be3f378576261ae3e\&tx_ttnews[any_of_the_words]=Antonio\%20Giustozzi $\& t x \_t$ tnews[tt_news] $=4889 \&$ tx_ttnews $[$ backPid] $=7 \& \mathrm{cHash}=$ bdbdea $632 \mathrm{a}$.
Die Ausbildungsunterstützung der NATO zielt auf die Ausbildung der Ausbilder und der militärischen Führer (mentoring). Die Durchführung der Ausbildung, die Planung und die Führung im Einsatz sollen in afghanischer Hand bleiben. In Gegensatz zur früheren Nationalarmee besteht das Offizierkorps nun hauptsächlich aus Tadschiken, gefolgt von den Paschtunen als größter Bevölkerungsgruppe. ${ }^{30}$ Rekruten erhalten zunächst an der zentralen Ausbildungseinrichtung in Kabul (KMTC) ihre infanteristische Grundausbildung (basic warrior training), die zehn Wochen dauert. Knapp ein Drittel der ausgebildeten Soldaten absolviert danach in weiteren sechs bis acht Wochen eine Spezialausbildung in einem von zehn Bereichen wie Artillerie oder Logistik. ${ }^{31}$ Jede ANA-Einheit wird anschließend von einem amerikanischen Team (Embedded Training Team, ETT) oder einem ISAF-Team (Operational Mentoring and Liaison Team, OMLT) betreut. Sie beraten die militärischen Führer, haben direkten Zugriff auf ISAF-Ressourcen und überprüfen kontinuierlich die Einheiten. Die Teams bestehen aus erfahrenen Offizieren und Unteroffizieren der NATO-Länder. Sie begleiten und beraten die afghanischen Soldaten im täglichen Dienst und im Einsatz, ermöglichen den Zugriff auf Luftunterstützung, Feuerverstärkung und medizinische Hilfe und koordinieren ihre Versorgung. Ihre Stationierung orientiert sich an den Standorten der zu betreuenden ANA-Einheiten und des Heeresfliegerkorps sowie der Ausbildungseinrichtungen und an der Unterstützung für den Generalstab und das Ministerium. Weitere Ausbildungshilfe besteht darin, den Kommandeuren und Abteilungsleitern beratend zur Seite zu stehen sowie Generäle und Stabsoffiziere etwa bei Inspektionen und Truppenbesuchen zu begleiten. So berät der deutsche Leiter der OMLTs beim 209. Korps den Kommandierenden General, Generalmajor Murad.

Nach wie vor gibt es zu wenig Ausbilder, ${ }^{32}$ die nach Meinung von Beobachtern noch dazu zu viele unterschiedliche Methoden anwenden. ${ }^{33}$ Die US-ETTs mit zwölf bis 16 Mann pro Team hatten im Juni 2009 anstelle 3.313 als erforderlich erachtete Ausbilder eine Gesamtstärke von 1.665 Mann. Allerdings soll diese Lücke durch die im Februar 2009 von Präsident Obama autorisierte Truppenverstärkung von über 21.000 Soldatinnen und Soldaten (davon 4.000 Ausbilder) ausgeglichen werden - dann soll erstmals seit 2002 die Trainingsmission voll besetzt sein. ${ }^{34}$ Die OMLTs der ISAF lehnen sich in ihrer Arbeit auf

30 Verlässliche Daten liegen nicht vor. Siehe Giustozzi, "Afghanistan's National Army" sowie ders., "Auxiliary Force or National Army?", S. 61. Vgl. Cordesman/Mausner/Kasten, Winning in Afghanistan, S. 55; Younossi u.a., The Long March, S. 21f.

31 Ali Ahmad, Briefing: Kabul Military Training Center (KMTC), 29. September 2008; TAG/KMTC Mentor Group/CSTC-A, Command Brief, Kabul 2008. Vgl. Younossi u.a., The Long March, S. 32

32 "Because the U.S. effort to build the ANSF has been an 'economy of force' operation, and, consequently, has not been adequately resourced with the appropriate number and skill sets of personnel, progress in creating the ANA and ANP has been less expeditious than it potentially could have been. Failure to provide the personnel resources the ANSF train and equip mission requires has delayed and will continue to delay building the sustainable capability of the ANSF to successfully fight this war, and, unless addressed, will prolong the duration of U.S. and Coalition/ISAF security responsibilities in Afghanistan." US Department of Defense Inspector General, Report On The Assessment of US And Coalition Plans To Train, Equip, And Field The Afghan National Security Forces Arlington/VA: Office of the Assistant Inspector General for Special Plans \& Operations 30 September 2009, S.37, http://www.dodig.mil/SPO/Reports/ SPO2009-007_final.pdf.

33 Siehe Chandra, "Making of the New Afghan National Army", S.58 sowie Cordesman/Mausner/Kasten, Winning in Afghanistan, S. $48 \mathrm{f}$ und S. 77ff.

34 Siehe Sydney J. Freedberg, "In Afghanistan, Training Up Is Hard To Do", National Journal, 24. Oktober 2009, http://www.nationaljournal.com/njmagazine/cs_20091024_5750.php?. 
Korps-, Brigade- und Kandakebene an das US-Konzept an - wie auch bei Ausbildung, taktischen Einsatzdoktrinen und logistischen Aufgaben. Die Doppelunterstellung unter die US-Streitkräfte (CSTC-A) und ISAF sorgte in der Vergangenheit für keine größeren Probleme, ${ }^{35}$ wurde inzwischen aber durch die NATOTrainingsmission (NTMA) abgelöst, die unter gemeinsamer Führung eines Generals der US-Armee steht. Nach NATO-Angaben werden im Dezember 201084 statt 56 OMLTs mit jeweils bis zu 30 Mann zur Truppenausbildung benötigt. Darüber hinaus hat die NATO 103 OMLTs zugesagt, sobald die ANA-Sollstärke von 134.000 erreicht ist - allerdings ist diese Planung noch am Zieldatum Dezember 2011 orientiert. ${ }^{36}$

Falls ein beschleunigter Aufwuchs bis Oktober 2010 erfolgen soll, sind zusätzliche Teams früher einzuplanen. ${ }^{37}$ McChrystal forderte zudem eine Truppenstärke von 240.000 Mann und Minister Wardak favorisierte in der Vergangenheit eine Zahl von 300.000 Mann. ${ }^{38}$ Um effektiv die Bevölkerung schützen zu können, wurde auch eine Erhöhung auf 325.000 Mann vorgeschlagen. ${ }^{39}$ Je höher das Ausbildungstempo, desto geringer ist jedoch die Qualität. Ausbildung nach dem Motto „Masse statt Klasse“ unterminiert Moral und Motivation. ${ }^{40}$ Die Folge kann sein - wie irakische Beispiele zeigen - dass sich nicht ausreichend ausgebildete und ausgerüstete Einheiten auflösen, sobald sie in Kampfeinsätze geraten. ${ }^{41}$ Als Achillesferse hat sich die Ausbildung afghanischer Offiziere und Unteroffiziere erwiesen; daher wurde vorgeschlagen, größere statt mehr Verbände zu schaffen und statt das Offizierskorps zu vergrößern, die vorhandenen militärischen Führer besser auszubilden. ${ }^{42}$

\subsection{Ausrüstung}

Die ANA sei schlechter ausgerüstet als die Mudschaheddin vor 20 Jahren, soll der afghanische Verteidigungsminister bemängelt haben. ${ }^{43}$ Tatsächlich sind Soldaten teilweise mit Sturmgewehren AK-47 („Kalaschnikow“) bewaffnet, außerdem stammen Geschütze und Panzer (T-55, T-62) noch aus der Sowjetzeit. Sukzessive eingeführt werden moderne, allerdings schwerer zu handhabende US-Gewehre der Typen M-16 und M-4 sowie Granatwerfer (M-203) und bis Mitte 2010 etwa

35 Vgl. Hans Hinrich Kühl, „Als Leiter eines Ausbildungsteams in Afghanistan“, BORDEAUXROT 1/2007, http://www.die-bordeauxroten.de/ebay/ArchivBR/082_BR2007_S5-10.pdf.

36 US Department of Defense, Progress toward Security and Stability in Afghanistan, S.6f und S.30; Cordesman, Afghan National Security Forces, S. 21; NATO, Afghanistan Report 2009, Büssel: NATO, o.J., S. 13.

37 Als Maximalforderung wurden 10.000-15.000 zusätzliche Ausbilder genannt. Siehe Thom Shanker/John H. Cushman, ,Reviews Raise Doubt on Training of Afghan Forces“, New York Times, 6. November 2009, S.A01.

38 Ann Scott Tyson/Walter Pincus, "U.S., NATO to Revamp Afghan Training Mission", WashP, 12. September 2009, http://www.washingtonpost.com/wpdyn/content/article/2009/09/11/AR2009091103954_pf.html.

39 Siehe Tony Capaccio, „U.S. Intelligence Head Says Afghan Army Must Grow to $325,000 "$ " Bloomberg, 6. August 2009, http://www.bloomberg.com/apps/ news?pid=20601091\&sid=awAx2W_1XFyc\#.

40 "Due to the significant challenges faced, the expansion of the ANA beyond the 134,000 objective could diminish force quality and its long-term viability, unless additional U.S. and international resources are made available well in advance." US Department of Defense, Report On the Assessment of US and Coalition Plans, S. 91

41 Freedberg, "In Afghanistan, Training Up Is Hard To Do" (Anm. 34)

42 Siehe Jeff Haynes, "Reforming the Afghan National Army", Security Watch, 25. November 2009, http://www.isn.ethz.ch/isn/Current-Affairs/SecurityWatch/Detail/?lng=en\&id=109959.

43 Chandra, "Making of the New Afghan National Army", S. 61.
4.100 gepanzerte Mehrzweckfahrzeuge des Typs „Humvee“ (High Mobility Multipurpose Wheeled Vehicle, HMMWV). Die Inbetriebnahme der Fahrzeuge ist jedoch mit zahlreichen Problemen belastet und verzögert sich. ${ }^{44}$ Moderne Waffen sind dabei weniger wichtig als Transportmittel, funktionierende Munition und angemessene Unterbringung - nur 24 von 144 Infrastrukturprojekten sind abgeschlossen. ${ }^{45}$

Um den Ausrüstungszustand zu verbessern, wurden Programme aufgelegt wie das „ANA Equipment Donation Programme“ zur Koordination der Ausrüstungsunterstützung sowie der „ANA Trust Fund“, der Betriebs-, Transport- und Installationskosten übernimmt, Ausrüstung finanziert und Auslandstrainings organisiert. Zudem kam militärisch-technische Ausbildungs- und/ oder Ausrüstungshilfe aus China, Russland und Indien sowie Iran und Jordanien. ${ }^{46}$

Zahlreiche Fälle der Unterschlagung von Ausrüstungsmaterial wie Winterbekleidung und mutwilliger Beschädigung von Fahrzeugen wurden dokumentiert. So musste Material mehrfach (300 Prozent) geliefert werden und 21 von 25 neuen Fahrzeugen wurden bei der Überführung zu einem Kandak beschädigt oder zerstört. Verantwortliche wurden nicht zur Rechenschaft gezogen. ${ }^{47}$ Zudem gelangte Ausrüstungshilfe auf den Schwarzmarkt - darunter „Hunderte deutsche Pistolen“, die 2005 aus Bundeswehrbeständen an ANA und ANP übergeben worden waren. ${ }^{48}$ Auch hier zeichnet sich ein langwieriger Lernprozess ab: Nicht nur muss Verantwortungsgefühl etabliert, sondern auch die grassierende Empfängermentalität („Wenn etwas kaputt geht, wird's die internationale Gemeinschaft schon richten“) ${ }^{49}$ überwunden werden. Die Ursachen dafür zu beseitigen - eine Kultur der Armut, entstanden in 30 Jahren unter fremder Besatzung, Bürgerkrieg und Unterdrückung - unterstreicht einmal mehr die Notwendigkeit ziviler Maßnahmen, um die Herausbildung eines bescheidenen Wohlstandes zu fördern. ${ }^{50}$

\subsection{Der deutsche Beitrag}

Deutschland unterstützt im Norden Afghanistans die Ausbildung des 209. ANA-Korps mit Hauptquartier in Mazar-e-Sharif. Im Jahre 2002 aufgestellt, verfügt das Korps über zwei Brigaden, die jeweils in Mazar-e-Sharif und Kundus stationiert sind. Vier von zehn Kandaks haben das höchste Fähigkeitsprofil (CM-1) erreicht. ${ }^{51}$

44 US Department of Defense, Progress toward Security and Stability in Afghanistan, S. 32 und S. 99.

45 Younossi u.a., The Long March, S. 25

46 Chandra, "Making of the New Afghan National Army", S. 62f; NATO, Afghanistan Report 2009, S. 14

47 US Department of Defense, Report On the Assessment of US and Coalition Plans, S. $57 \mathrm{f}$.

48 Über 200.000 Gewehre, Pistolen und andere Waffen wurden übergeben, ohne deren Verbleib zu erfassen. Siehe Eric Schmitt, „Afghan arms are at risk, report says“, International Herald Tribune 12. Februar 2009; „Deutsche Pistolen auf dem Basar“, Frankfurter Allgemeine Zeitung, 13. Oktober 2009, S. 2.

49 Theo Sommer, „Afghanistan, wie lange noch?“, Die Zeit, Nr. 44 (23. Oktober 2008), S. 7

50 "U.S. trainers reported that the mindset of not wanting to give up supply items for fear that no more would follow was pervasive throughout the ANSF supply system." US Department of Defense, Report On The Assessment of US And Coalition Plans, S. 32

51 Vgl. im Folgenden Paul, (Wieder-) Aufbau der Afghanischen Nationalarmee, S. 6-7. 
Der Beitrag des Bundesverteidigungsministeriums konzentriert sich vor allem darauf, die Ausbildung von Truppe und Stäben zu unterstützen, ihnen bei der Planung taktischer Operationen zu helfen und sie im Einsatz zu begleiten. Dies umfasst Checkpoints und Patrouillen sowie Präsenz in der Fläche, des Weiteren illegale Waffenlager aufzuspüren, terroristische Gruppierungen zu bekämpfen, Entwaffnungen und Durchsuchungen vorzunehmen, gezielt Personen festzusetzen, wichtige Einrichtungen zu schützen sowie Veranstaltungen und gefährdete Personen abzusichern. Darüber hinaus ist Deutschland als Leitnation und Finanzträger für die ANA-Logistikschule in Kabul mit 25 Mentoren und die ANA-Pionierschule in Mazar-e-Sharif mit 18 Mentoren aktiv.

Im August 2007 unterstützten 55 Bundeswehrsoldaten die Ausbildung von 4.000 ANA-Soldaten des 209. Korps. Zwei Jahre später waren 300 Bundeswehrsoldaten in sieben OMLTs für 8.000 (von insgesamt 67.000) ANA-Soldaten zuständig. Um die angestrebte Personalstärke von landesweit 134.000 Mann (davon 12.500 in der Nordregion) zu erreichen, sollen im Juli 2010 insgesamt 370 deutsche Soldaten als Ausbilder in zehn OMLTs tätig sein. Soll der Aufwuchs bereits bis Oktober 2010 erfolgen, dürfte dies planerische und praktische Probleme bereiten. Im Oktober 2009 waren alle deutschen OMLTs vollzählig.

Die Soldatinnen und Soldaten der Bundeswehr sind in Ausübung ihres Auftrags an die deutschen Einsatzregeln gebunden - so an die grundsätzliche geografische Festlegung auf Nordafghanistan und Kabul. Der Einsatz einer ANA-Einheit außerhalb dieser Region wäre für Ausbilder und Truppe daher gleichermaßen schwierig, weil deutsche OMLT-Ausbilder die afghanischen Soldaten mitten in ihrer Ausbildung alleine lassen müssten. Ausnahmen sind - nach ministerieller Genehmigung - aber möglich. Experten der Bundeswehr erarbeiten derzeit ein neues Ausbildungskonzept: Als denkbar gelte ein bisher von der Bundeswehr nicht praktiziertes Prinzip, wonach die Ausbildung im konkreten Einsatz außerhalb der Kasernengelände erfolgen könne. ${ }^{52}$ Auch die übliche Kontingentdauer der Bundeswehr von vier Monaten kann verlängert werden, um innerhalb des ANA-Ausbildungszyklus von neun Monaten (zwei Monate Training, sechs Monate Einsatz, ein Monat Fortbildung/Pause) $)^{53}$ ANA-Stäbe und Truppen fortwährend zu betreuen.

Nach den Vorstellungen McChrystals sollen künftig alle Operationen von ISAF und ANA "gemischt" erfolgen (embedded partnering). In diesem vertieften Ansatz gemeinsamer Operationsführung soll es keine „getrennten Operationen“ mehr geben. Sollte dies umgesetzt werden, könnten Ausnahmegenehmigungen zur Regel zu werden. Auch wären mehr Soldaten sowie bereits knappe Ressourcen wie Logistik, Sanitäter und Hubschrauber nötig. ${ }^{54}$ Dabei ist die Bundeswehr unmittelbar von den seit 2007 wachsenden Aktivitäten der Taliban betroffen. Sie haben Kundus als ehemalige Taliban-Hochburg zum Ausgangspunkt für Angriffe gegen die Nachschublinien der NATO (via Russland und Zentralasien) in Nordafghanistan

52 Vgl. „Westerwelle blockiert Guttenbergs Pläne“, Zeit Online, 12. Januar 2010, http://www.zeit.de/politik/deutschland/2010-01/westerwelle-afghanistantruppenaufstockung.

53 Siehe Younossi u.a., The Long March, S. 39.

54 Vgl. Rainer Glatz, „Eine rein militärische Aufstockung wird nichts nützen“, Tagesspiegel, 24.10.09, S. 5. gewählt und sind damit auch der Aufstandsbekämpfung im Süden ausgewichen. ${ }^{55}$

\section{Die ANA im Einsatz}

Seit dem ersten größeren Einsatz im Juli 2003 finden zunehmend Operationen unter afghanischer Führung statt; im Jahr 2008 waren es 61 Prozent der Kampfeinsätze. ${ }^{56}$ Die ANA ist in der Lage, Operationen bis zur Kompanieebene (etwa 120 Mann) selbst zu leiten. ${ }^{57}$

Auf den ersten Blick mag es paradox erscheinen, dass die wachsende Zahl von ANA-Soldaten mitverantwortlich dafür ist, dass es seit 2008 zu einem Anstieg der Kampfhandlungen kam. Aber durch Kandaks der Kategorie CM-1 wurde eine Offensive in Gegenden möglich, die unter die Kontrolle der Taliban geraten waren. Daher könnte sich die Gewalt noch steigern, wenn ANA-Truppen in die paschtunischen Siedlungsgebiete im Süden und Osten vorrücken (und dabei einen Verdrängungseffekt nach Norden auslösen). Dies könnte zu einer Radikalisierung der friedlichen Mehrheit der Paschtunen (als größter Bevölkerungsgruppe) und schließlich zu einer Solidarisierung mit den Taliban führen. Das Gewaltpotenzial ist beträchtlich: Nicht jeder Paschtune ist ein Talib, aber die meisten Taliban sind Paschtunen. Und über 70 Prozent der Bevölkerung ist jünger als 25 Jahre. Dies zeigt einmal mehr, dass eine Konzentration auf militärische Aspekte zu kurz greift und zivile Aufbauarbeit - Staatsaufbau ebenso wie Förderung von Handwerk und Landwirtschaft - im Vordergrund stehen muss. Diese benötigt jedoch ein sicheres und stabiles Umfeld. In diesem Sinne bildet die Ausbildungsunterstützung der ANA ein ergänzendes Element in einem zivile und militärische Aspekte umfassenden Ansatz.

\section{Perspektiven}

Die ANA wird in einem überschaubaren Zeitraum nicht in der Lage sein, eigenständig Sicherheit in Afghanistan zu gewährleisten. Sie ist weiterhin abhängig von den Ausbilderteams, die oberhalb der Kompanieebene häufig als militärische Führer fungieren, und damit verbundenen militärischen Fähigkeiten wie Luftunterstützung und Logistik. Zwar wird ein eigenes Heeresfliegerkorps aufgebaut, doch nach Expertenmeinung wird die ANA bis mindestens 2016 auf Luftnahunterstützung angewiesen sein. ${ }^{58}$ Zwar ruhen die Hoffnungen der Bevölkerung auf der ANA (was auch die jüngste Umfrage bestätigt), ihr Wiederaufbau kann aber noch nicht als Erfolgsgeschichte bezeichnet werden. Erst wenn sie selbstständig und verantwortlich handeln kann, ist ein wichtiges Erfolgskriterium dafür erfüllt, die

55 Vgl. Carlotta Gall, "Taliban Open Northern Front in Afghanistan”, New York Times, 26. November 2009, http://www.nytimes.com/2009/11/27/world/asia/ 27kunduz.html.

56 Cordesman/Mausner/Kasten, Winning in Afghanistan, S. 34 sowie Angaben gemäß ANA-Website (Stand April 2007), http://www.mod.gov.af/\#Training. Vgl. Giustozzi, "Auxiliary Force or National Army?, S. 54ff.

57 Siehe Gilles Dorronsoro, „Filling the Void: Dispatch from Afghanistan”, Web Commentary, 26. August 2009, http://carnegieendowment.org/publications/ index.cfm?fa=view\&id=23718\&prog=zgp\&proj=zsa.

58 Younossi u.a., The Long March, S.26; NATO, Afghanistan Report 2009, S. 15. 
ISAF-Streitkräfte zu reduzieren. Das reicht aber noch nicht aus und ist auch nicht mit selbsttragender Sicherheit gleichzusetzen.

Wird Afghanistan jemals imstande sein, seine Bevölkerung zu ernähren und neu geschaffene staatliche Strukturen - mit dem notwendigen Personal, also auch mit Sicherheitskräften - zu bezahlen? Wie im Falle der Polizei besteht die Gefahr, dass Soldaten zu den durch Einnahmen aus dem Drogengeschäft gut ausgestatteten Milizen oder gar zu den Taliban abwandern. Stammeskrieger, die einem archaischen Ehrenkodex verhaftet sind, können nicht kurzerhand zu Staatsbürgern in Uniform verwandelt werden - zumal, wenn ein Staatswesen nur rudimentär vorhanden ist und Uniformen fehlen. Die Wiedereinführung einer allgemeinen Wehrpflicht ${ }^{59}$ wäre eine kostengünstige Alternative und dürfte auch von der Bevölkerung unterstützt werden. Die Afghanen verbinden mit der hoch angesehenen Armee gewissermaßen einen zivilisierenden Einfluss, was auch die Loyalität zum Zentralstaat befördern könnte.

Die internationale Gemeinschaft wird noch einige Jahre und erhebliche Mittel investieren müssen, bevor selbsttragende Sicherheit geschaffen ist. Die Skepsis wird von führenden Offizieren der Bundeswehr geteilt. Zwar halten es einige für möglich,

59 Vgl. Ronald E. Neumann, „Afghans, Report for Duty“, New York Times, 14. Januar 2008, S. A25. in einzelnen Distrikten im Norden die Verantwortung für die Sicherheit afghanischen Kräften zu übertragen. Doch weisen andere darauf hin, dass dies wenig nütze, wenn in den angrenzenden Gebieten weiter Unsicherheit und Gewalt herrschten. Außerdem müssten weiterhin ausländische Kräfte vorgehalten werden, um die Dauerhaftigkeit solch nationaler Lösungen zu gewährleisten. ${ }^{60}$ Schließlich bedarf eine partnerschaftliche Beziehung einer Ausbildung auf Augenhöhe und in angemessenem Tempo, um ANA-Soldaten auch mental aus der Rolle einer Hilfstruppe zu emanzipieren: An Afghan face needs an Afghan pace. Dazu bedarf es strategischer Geduld, politischen Durchhaltevermögens und vorbehaltloser Aufklärung der Bevölkerung in Afghanistan und den ISAF-Entsendestaaten über die Chancen und Risiken des Einsatzes. Die ANA ist auf dem richtigen, wenn auch mühsamen Weg zum Erfolg. Derzeit aber kommt das von NATO-Generalsekretär Anders Fogh Rasmussen apostrophierte Licht am Ende des Tunnels ${ }^{61}$ noch aus den Taschenlampen der eigenen Ausbilder.

60 Siehe Peter Blechschmidt, "Afghanistans erfolglose Armee", Süddeutsche Zeitung, 18. November 2009, S. 1.

61 Anders Fogh Rasmussen, „Afghanistan and NATO: The Way Forward”. Speech by NATO Secretary General at the Atlantic Council of the United States, 28. September 2009, http://www.nato.int/cps/en/natolive/opinions_57722. htm.

\title{
Das Verbot von Chemiewaffen: Fünf Hürden auf dem Weg zu einer chemiewaffenfreien Welt
}

\author{
Thomas Müller-Färber* und Roland Hiemann**
}

\begin{abstract}
This article focuses on the essential challenges to the idea of a world free of chemical weapons. The authors identify five different problem areas carrying the potential to substantially reduce the feasibility of the global prohibition of chemical weapons: the delayed demilitarisation of chemical weapons, related capabilities of non-member states to the Chemical Weapons Convention, the spread and development of non-lethal chemical weapons, the accelerated scientific and technology progress in biochemical sciences and, finally, weak public support for the elimination of chemical weapons. Notwithstanding the substantial progress made in the past, it is argued that the international community needs to tackle these pivotal obstacles in the mid- and long term future in order to achieve a world without chemical weapons.
\end{abstract}

Keywords: Disarmament, chemical weapons, Chemical Weapons Convention (CWC), proliferation Abrüstung, Chemiewaffen, Chemiewaffen-Übereinkommen (CWÜ), Verbreitung

\section{Einführung}

Spätestens seit der Prager Rede von US-Präsident Barack Obama im April 2009, in der er sich für eine Welt ohne Atomwaffen aussprach, hat die Idee von „Global Zero“ verstärkte Aufmerksamkeit erhalten. ${ }^{1}$ Seitdem beherrscht der Gedanke an eine

* Thomas Müller-Färber ist Stipendiat der Friedrich-Ebert-Stiftung und Doktorand an der Eberhard-Karls-Universität Tübingen.

** Roland Hiemann ist Doktorand und Stipendiat der Stiftung Wissenschaft und Politik (SWP) in Berlin.

1 Vgl. Remarks of President Barack Obama (Excerpts), in: Arms Control Today, Mai 2009, http://www.armscontrol.org/node/3644, abgerufen am 19.11.2009.
Welt frei von Kernwaffen viele aktuelle rüstungs- und sicherheitspolitische Debatten. Während sich namhafte Experten in jüngster Vergangenheit mit der Frage auseinandergesetzt haben, wie die Vision einer vollständigen nuklearen Abrüstung gelingen könnte, ${ }^{2}$ ist die „Global Zero“-Idee für Chemiewaffen (CW) bereits in vielerlei Hinsicht Wirklichkeit geworden. CW sind heute mit einem umfassenden, nahezu weltweit gültigen

2 Vgl. Shultz, George P.; Perry, William J.; Kissinger, Henry A; Nunn, Sam: A World Free of Nuclear Weapons, The Wall Street Journal, 04. Januar 2007 / Schmidt, Helmut; Weizsäcker, Richard von; Bahr, Egon; Genscher, Hans-Dietrich: Für eine atomwaffenfreie Welt, Frankfurter Allgemeine Zeitung, 09. Januar 2009. 